\title{
Off-shell Higgs signal and total width determination at the LHC
}

\section{Nikolas Kauer*}

Department of Physics, Royal Holloway, University of London, Egham TW20 OEX, UK

E-mail: n.kauererhul.ac.uk

\begin{abstract}
A substantial off-shell Higgs boson signal in the gluon fusion and vector boson fusion $H \rightarrow Z Z$ and $H \rightarrow W W$ channels at the Large Hadron Collider (LHC) facilitates a novel, complementary approach to constraining the total Higgs width $\Gamma_{H}$. With LHC Run 1 data, experimental analyses by CMS and ATLAS find $\Gamma_{H}<5.4 \Gamma_{H}^{\mathrm{SM}}$ and $\Gamma_{H}<[4.5,7.5] \Gamma_{H}^{\mathrm{SM}}$ at $95 \%$ confidence level, respectively, where $\Gamma_{H}^{\mathrm{SM}}$ is the expected value in the Standard Model at the measured Higgs boson mass. I review the theoretical basis of the new approach and discuss its significance in comparison to other methods to bound and measure the Higgs width at the LHC and future colliders.
\end{abstract}

Frontiers of Fundamental Physics 14

15-18 July 2014

Aix Marseille University (AMU) Saint-Charles Campus, Marseille, France

*Speaker. 


\section{Introduction}

The fundamental particle predicted by the Standard Model (SM) Higgs mechanism [1], i.e. the Higgs boson, was discovered at the LHC in 2012 [2]. A thorough examination has since taken place and its properties have been found to be in agreement with theoretical expectations. No compelling deviations from the SM have been discovered so far. An important property of the Higgs boson is its total decay width, with a predicted $\mathrm{SM}$ value of $\Gamma_{H}^{\mathrm{SM}} \approx 4 \mathrm{MeV}$, which is more than two orders of magnitude smaller than the experimental Higgs mass resolution at the LHC, which is of order $1 \mathrm{GeV}$. At the LHC, any direct Higgs width measurement via the resonance shape is thus limited to an uncertainty of $\Delta \Gamma_{H} \sim 1 \mathrm{GeV}$. ${ }^{1}$ Since the resonant ("on-peak") Higgs cross section depends on $\Gamma_{H}$, the Higgs couplings and width cannot be determined independently at the LHC without relying on theoretical assumptions [4,5]. For instance, in models without triplett or higher $S U$ (2) representations an upper limit for the $H W W$ or $H Z Z$ coupling exists and an upper bound for the Higgs width can be obtained that is of the order of the SM Higgs width [6,7]. Assuming no beyond-SM (BSM) Higgs decays, and suggestive Higgs coupling parameterisations, one can fit the Higgs width to data and finds similar agreement with $\Gamma_{H}^{\mathrm{SM}}$ [8-12]. At a future $e^{+} e^{-}$collider, a largely model-independent indirect determination of the Higgs width will be possible, with a predicted accuracy of 5-10\% at the International Linear Collider [12-14]. A future muon collider could permit a direct Higgs width measurement via threshold scan with an estimated accuracy of 4-9\% [15]. Two novel, complementary methods to constrain the Higgs width at the LHC are reviewed in Section 2.

\section{Off-shell Higgs signal enabled total width determination at the LHC}

The existence of a substantial off-shell Higgs boson signal in the gluon fusion $H \rightarrow Z Z$ and $H \rightarrow W W$ channels at the LHC was first pointed out in Ref. [16]. ${ }^{2}$ In Fig. 1, representative graphs for the Higgs signal and continuum background processes are shown as well as $M_{V V}$ distributions that show the enhanced off-shell Higgs signal, which constitutes an $\mathscr{O}(5-10 \%)$ correction to inclusive $g g \rightarrow H \rightarrow V V$ production in narrow-width approximation (NWA). With typical experimental LHC selection cuts this correction increases to $\mathscr{O}(10-20 \%)$. Also shown in Fig. 1 is the sizeable signal-background interference in the off-shell region, which facilitates unitarity at high energies and has been calculated in Refs. [16,18-25]. Note that the interfering $g g \rightarrow V V$ continuum background at LO is formally part of the NNLO corrections to $p p \rightarrow V V[26,27]$.

A proposal to exploit the Higgs width independence of the off-shell Higgs signal in order to break the NWA scaling degeneracy

$$
\sigma_{i \rightarrow H \rightarrow f} \stackrel{\text { NWA }}{\propto} \frac{g_{i}^{2} g_{f}^{2}}{\Gamma_{H}}, \quad \sigma \text { invariant if } g_{i} \rightarrow \xi g_{i}, g_{f} \rightarrow \xi g_{f}, \quad \Gamma_{H} \rightarrow \xi^{4} \Gamma_{H}
$$

of the on-peak Higgs signal in $g g \rightarrow H \rightarrow Z Z \rightarrow 4 \ell$ was first made in Ref. [28], which also provided a proof-of-concept phenomenological analysis which suggested that Higgs width constraints of $\Gamma_{H}<[20,38] \Gamma_{H}^{\mathrm{SM}}$ are feasible. A more detailed phenomenological analysis was subsequently

\footnotetext{
${ }^{1}$ For instance, Ref. [3] finds $\Gamma_{H}<3.4 \mathrm{GeV}$ at $95 \%$ confidence level (CL).

${ }^{2}$ The significance of the off-shell $H \rightarrow V V$ signal at a linear collider is discussed in Ref. [17].
} 

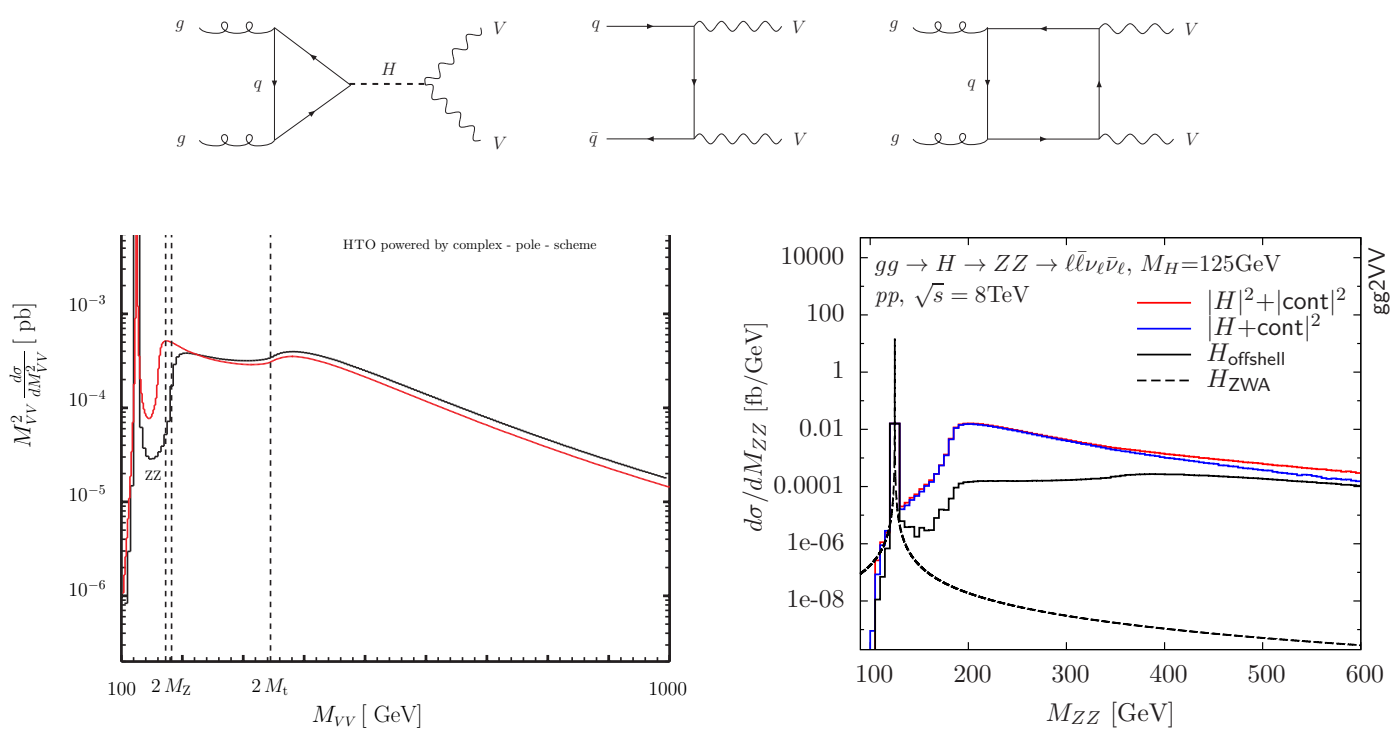

Figure 1: Representative Feynman graphs for the $g g \rightarrow H \rightarrow V V$ signal process (left) and the $q \bar{q}$ - (centre) and $g g$-initiated (right) continuum background processes at LO as well as $M_{V V}$ distributions that show the enhanced off-shell Higgs signal and sizeable Higgs-continuum interference (from [16]).
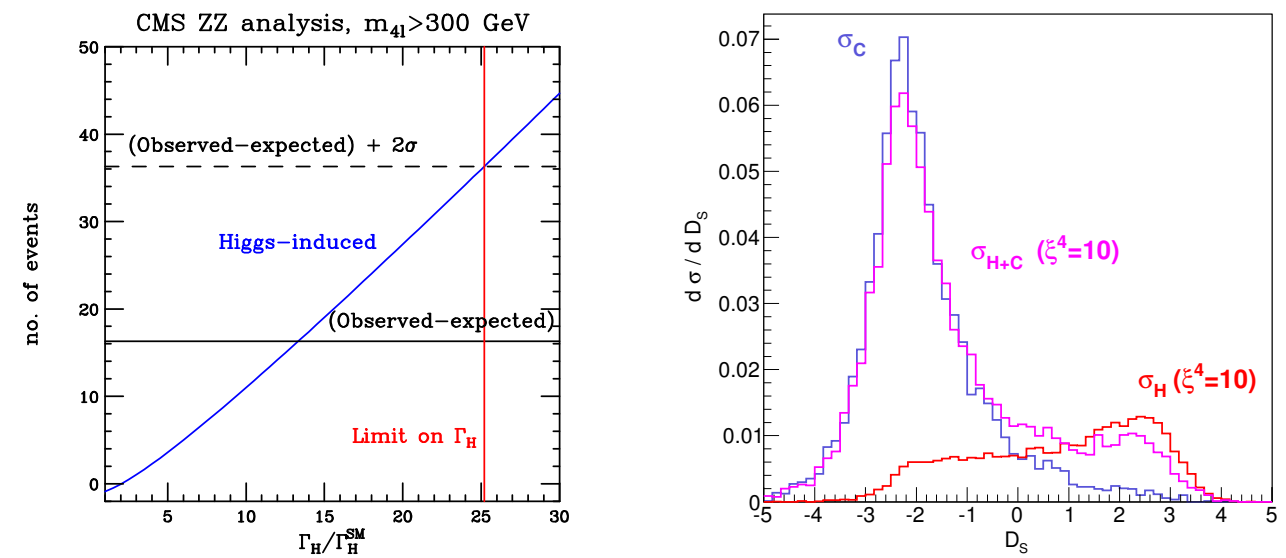

Figure 2: Results of a detailed phenomenological study of the off-shell Higgs width constraint approach (from [29]).

carried out in Refs. [29,30], which optimized the sensitivity of the method by exploiting the full differential cross section information using the Matrix Element Method [31] (see Fig. 2). After these phenomenological studies, CMS [32] (see Fig. 3) and ATLAS [33] (see Fig. 4) carried out full experimental simulations which also took into account the $2 \ell 2 v$ final state, vector boson fusion contributions and higher-order corrections. CMS and ATLAS thus found $\Gamma_{H}<5.4 \Gamma_{H}^{\mathrm{SM}}$ and $\Gamma_{H}<[4.5,7.5] \Gamma_{H}^{\mathrm{SM}}$ at $95 \% \mathrm{CL}$, respectively. More recently, theorists have demonstrated that the $Z Z+$ jet channel can be used to improve the obtained constraints [34]. That the off-shell Higgs width constraint approach is a priori model dependent was first pointed out in Ref. [35] and has been further studied in Refs. [36,37]. 

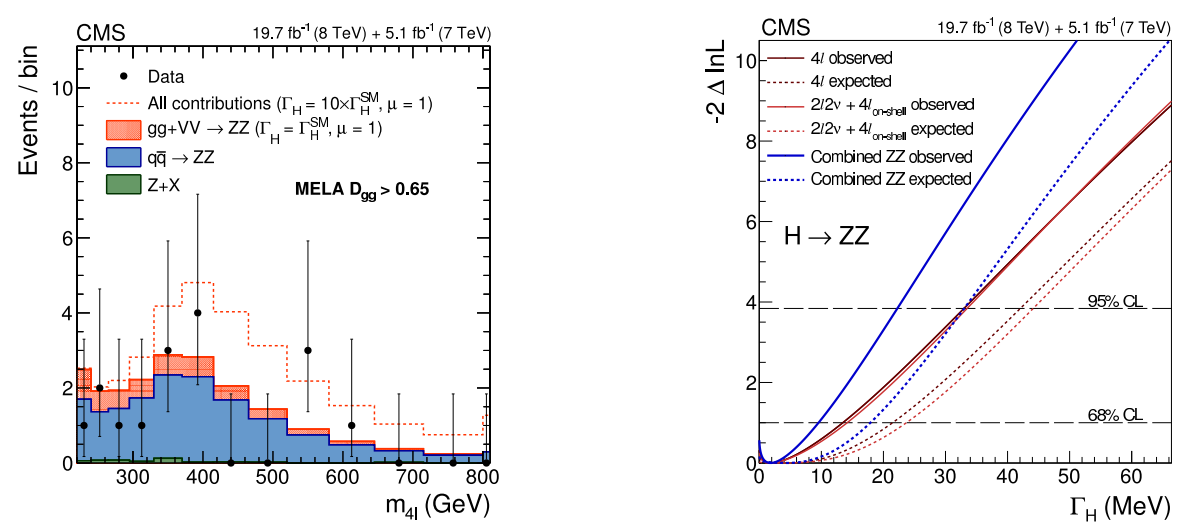

Figure 3: CMS study of off-shell Higgs width constraint approach (from [32]).
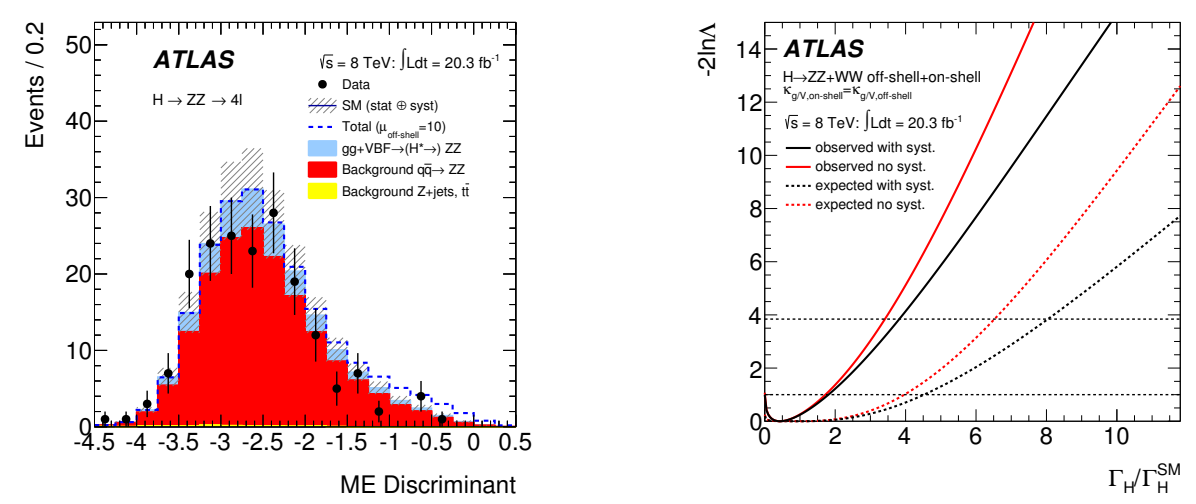

Figure 4: ATLAS study of off-shell Higgs width constraint approach (from [33]).

More generally, the off-shell $H \rightarrow V V$ signal can be used to disentangle degeneracies in parametric BSM studies or constrain higher dimensional operators in effective field theory (EFT) studies [36,38-43]. For instance, Refs. [40,41,43] analyse SM deviations of the effective $g g H$ and $H t \bar{t}$ coupling strengths in an EFT approach:

$$
\begin{aligned}
\mathscr{L} & =-c_{t} \frac{m_{t}}{v} \bar{t} t h+\frac{g_{s}^{2}}{48 \pi^{2}} c_{g} \frac{h}{v} G_{\mu v} G^{\mu v} \\
\mathscr{M}_{g g \rightarrow Z Z} & =\mathscr{M}_{h}+\mathscr{M}_{b k g}=c_{t} \mathscr{M}_{c_{t}}+c_{g} \mathscr{M}_{c_{g}}+\mathscr{M}_{b k g}
\end{aligned}
$$

One has: $\sigma \sim\left|c_{t}+c_{g}\right|^{2}$. The on-peak degeneracy $c_{t}+c_{g}=$ const is broken by off-shell data. Results of Ref. [41] are shown in Fig. 5.

An alternative method to constrain the total Higgs width was proposed in Ref. [44]. It exploits a sizeable asymmetric signal-background interference in the $g g \rightarrow H \rightarrow \gamma \gamma$ channel at the LHC, which was first pointed out and calculated at LO in Ref. [45]. ${ }^{3}$ A NLO calculation and analysis was carried out in Ref. [44] (see Fig. 6). This method is expected to yield competitive Higgs width constraints with $3 \mathrm{ab}^{-1}$ of LHC14 data.

\footnotetext{
${ }^{3}$ Signal-background interference and mass peak shift effects in the $q g$ and $q \bar{q}$ channels have been analysed at LO in Refs. [46,47].
} 


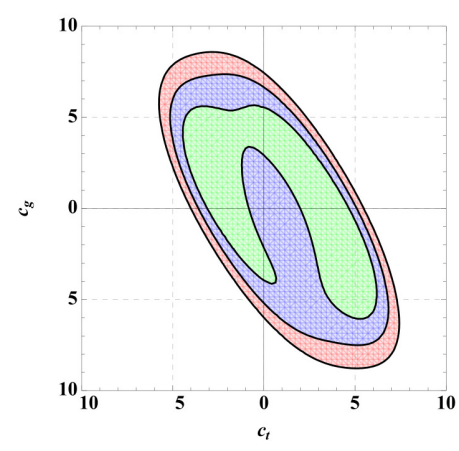

LHC 8 TeV CMS data

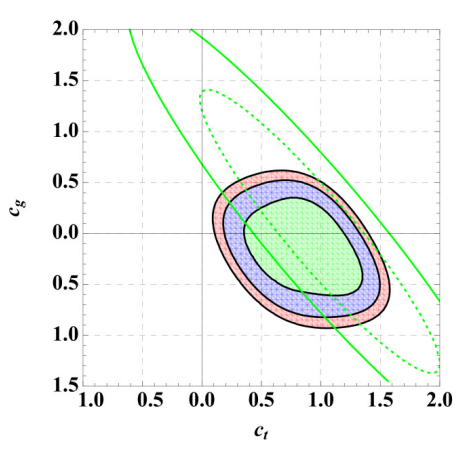

LHC $14 \mathrm{TeV} 3 \mathrm{ab}^{-1}$ data

Figure 5: Constraints in $\left(c_{t}, c_{g}\right)$ plane: $68 \%, 95 \%$ and $99 \%$ probability contours are shown (from [41]).
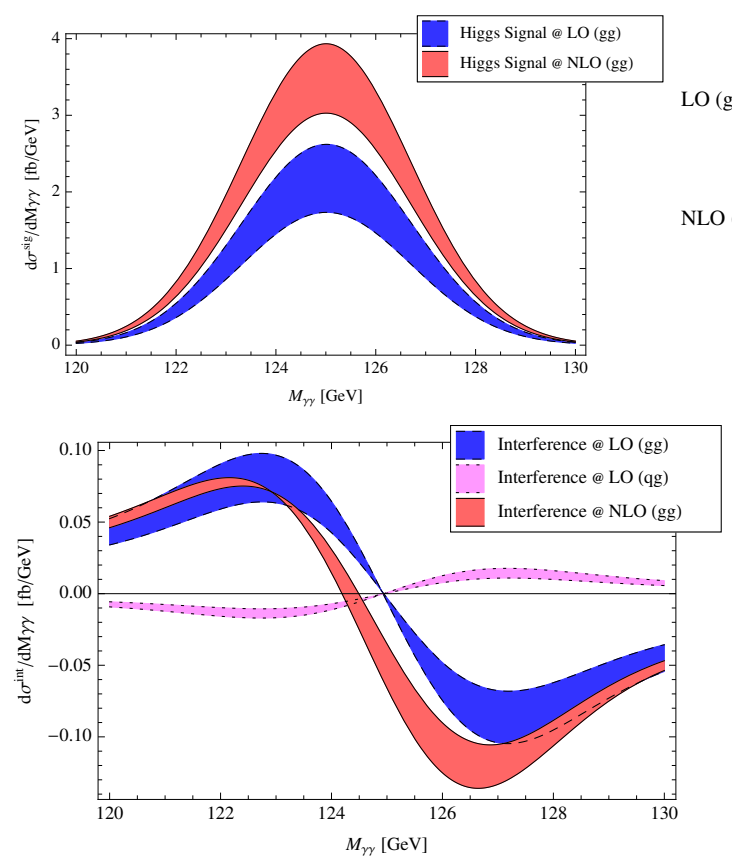
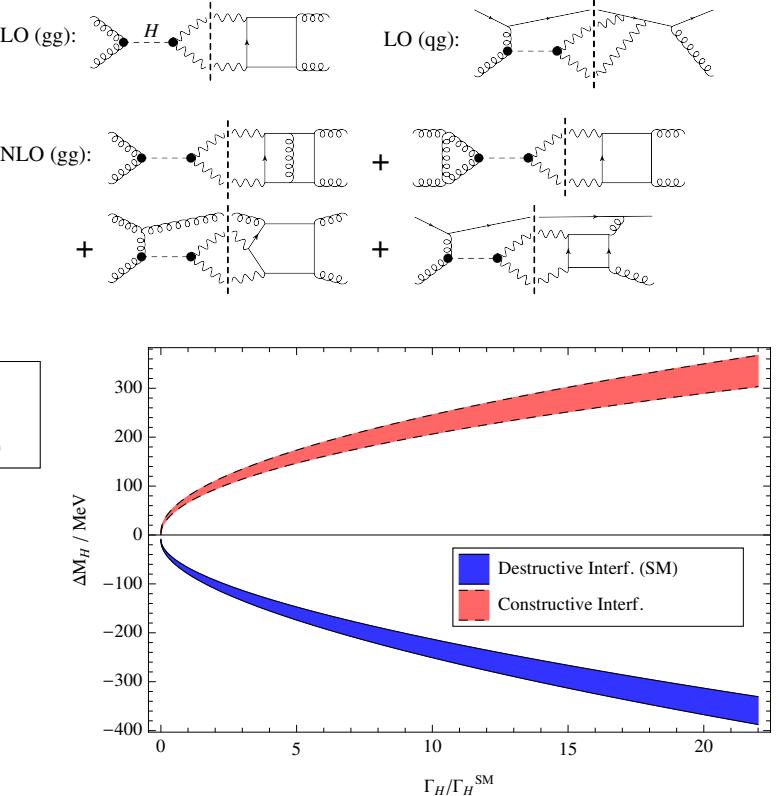

Figure 6: Signal-background interference enabled Higgs width constraints in $g g \rightarrow H \rightarrow \gamma \gamma$ (from [44])

\section{Conclusions}

Two novel, complementary methods to constrain the total Higgs width at the LHC have been reviewed. The first method relies on the experimental sensitivity to the Higgs-width-independent off-shell signal cross section in the $g g \rightarrow H \rightarrow V V$ channels, and with LHC Run 1 data yields a Higgs width constraint of $\Gamma_{H} \lesssim 5 \Gamma_{H}^{\mathrm{SM}}$. The second method relies on a sizeable asymmetric signalbackground interference in the $g g \rightarrow H \rightarrow \gamma \gamma$ channel that results in a Higgs mass peak shift which is expected to yield competitive Higgs width constraints with $3 \mathrm{ab}^{-1}$ of LHC14 data. 


\section{Acknowledgments}

Fruitful collaboration with G. Passarino and informative and useful discussions with A. Banfi, F. Caola, C. Charlot, R. Covarelli, S. Diglio, B. Di Micco, M. Dührssen, C. Englert, S. Forte, Y. Gao, S. Gascon-Shotkin, D. Gonçalves, M. Grazzini, C. Grojean, S. Heinemeyer, G. Isidori, S. Jäger, S. Liebler, H. Logan, I. Low, F. Maltoni, C. Mariotti, S. Marzani, M. Mühlleitner, B. Murray, C. O’Brien, C. Oleari, T. Plehn, M. Spannowsky, R. Tanaka, E. Vryonidou, J. Wang and G. Weiglein are gratefully acknowledged. I would like to thank the Galileo Galilei Institute for Theoretical Physics for hospitality and the INFN for partial support during the preparation of this paper. This work was supported by STFC grants ST/J000485/1, ST/J005010/1 and ST/L000512/1.

\section{References}

[1] P. W. Higgs, Broken symmetries, massless particles and gauge fields, Phys. Lett. 12 (1964) 132; P. W. Higgs, Broken symmetries and the masses of gauge bosons, Phys. Rev. Lett. 13 (1964) 508; P. W. Higgs, Spontaneous symmetry breakdown without massless bosons, Phys. Rev. 145 (1966) 1156; F. Englert and R. Brout, Broken symmetry and the mass of gauge vector mesons, Phys. Rev. Lett. 13 (1964) 321; G. S. Guralnik, C. R. Hagen and T. W. B. Kibble, Global conservation laws and massless particles, Phys. Rev. Lett. 13 (1964) 585.

[2] G. Aad et al. (ATLAS Collaboration), Observation of a new particle in the search for the Standard Model Higgs boson with the ATLAS detector at the LHC, Phys. Lett. B 716 (2012) 1 [arXiv:1207.7214 [hep-ex]]; S. Chatrchyan et al. (CMS Collaboration), Observation of a new boson at a mass of 125 GeV with the CMS experiment at the LHC, Phys. Lett. B 716 (2012) 30 [arXiv:1207.7235 [hep-ex]].

[3] S. Chatrchyan et al. (CMS Collaboration), Measurement of the properties of a Higgs boson in the four-lepton final state, Phys. Rev. D 89 (2014) 9, 092007 [arXiv:1312.5353 [hep-ex]].

[4] M. Duhrssen, S. Heinemeyer, H. Logan, D. Rainwater, G. Weiglein and D. Zeppenfeld, Extracting Higgs boson couplings from CERN LHC data, Phys. Rev. D 70 (2004) 113009 [hep-ph/0406323].

[5] S. Dittmaier, C. Mariotti, G. Passarino, R. Tanaka, S. Alekhin, J. Alwall, E. A. Bagnaschi et al., arXiv:1201.3084 [hep-ph].

[6] M. E. Peskin, Comparison of LHC and ILC Capabilities for Higgs Boson Coupling Measurements, arXiv:1207.2516 [hep-ph].

[7] B. A. Dobrescu and J. D. Lykken, Coupling spans of the Higgs-like boson, JHEP 1302 (2013) 073 [arXiv:1210.3342 [hep-ph]].

[8] V. Barger, M. Ishida and W. Y. Keung, Total Width of 125 GeV Higgs Boson, Phys. Rev. Lett. 108 (2012) 261801 [arXiv:1203.3456 [hep-ph]].

[9] K. Cheung, J. S. Lee and P. Y. Tseng, Higgs Precision (Higgcision) Era begins, JHEP 1305 (2013) 134 [arXiv:1302.3794 [hep-ph]].

[10] J. Ellis and T. You, Updated Global Analysis of Higgs Couplings, JHEP 1306 (2013) 103 [arXiv:1303.3879 [hep-ph]].

[11] A. Djouadi and G. Moreau, The couplings of the Higgs boson and its CP properties from fits of the signal strengths and their ratios at the 7+8 TeV LHC, Eur. Phys. J. C 73 (2013) 9, 2512 [arXiv:1303.6591 [hep-ph]]. 
[12] P. Bechtle, S. Heinemeyer, O. Stal, T. Stefaniak and G. Weiglein, Probing the Standard Model with Higgs signal rates from the Tevatron, the LHC and a future ILC, JHEP 1411 (2014) 039 [arXiv:1403.1582 [hep-ph]].

[13] T. Han, Z. Liu and J. Sayre, Potential Precision on Higgs Couplings and Total Width at the ILC, Phys. Rev. D 89 (2014) 11, 113006 [arXiv:1311.7155 [hep-ph]].

[14] M. E. Peskin, Estimation of LHC and ILC Capabilities for Precision Higgs Boson Coupling Measurements, arXiv:1312.4974 [hep-ph].

[15] T. Han and Z. Liu, Potential precision of a direct measurement of the Higgs boson total width at a muon collider, Phys. Rev. D 87 (2013) 3, 033007 [arXiv:1210.7803 [hep-ph]].

[16] N. Kauer and G. Passarino, Inadequacy of zero-width approximation for a light Higgs boson signal, JHEP 1208 (2012) 116 [arXiv:1206.4803 [hep-ph]].

[17] S. Liebler, G. Moortgat-Pick and G. Weiglein, Off-shell effects in Higgs processes at a linear collider and implications for the LHC, arXiv:1502.07970 [hep-ph].

[18] E. W. N. Glover and J. J. van der Bij, Vector boson pair production via gluon fusion, Phys. Lett. B 219 (1989) 488.

[19] E. W. N. Glover and J. J. van der Bij, Z-boson pair production via gluon fusion, Nucl. Phys. B 321 (1989) 561.

[20] T. Binoth, M. Ciccolini, N. Kauer and M. Kramer, Gluon-induced W-boson pair production at the LHC, JHEP 0612 (2006) 046 [hep-ph/0611170].

[21] J. M. Campbell, R. K. Ellis and C. Williams, Gluon-gluon contributions to $W^{+} W^{-}$production and Higgs interference effects, JHEP 1110 (2011) 005 [arXiv:1107.5569 [hep-ph]].

[22] M. Bonvini, F. Caola, S. Forte, K. Melnikov and G. Ridolfi, Signal-background interference effects for $g g \rightarrow H \rightarrow W^{+} W^{-}$beyond leading order, Phys. Rev. D 88 (2013) 3, 034032 [arXiv:1304.3053 [hep-ph]].

[23] N. Kauer, Interference effects for $H \rightarrow W W / Z Z \rightarrow \ell \bar{v}_{\ell} \bar{\ell} v_{\ell}$ searches in gluon fusion at the LHC, JHEP 1312 (2013) 082 [arXiv:1310.7011 [hep-ph]].

[24] J. M. Campbell, R. K. Ellis and C. Williams, Bounding the Higgs width at the LHC, PoS LL 2014 (2014) 008 [arXiv:1408.1723 [hep-ph]].

[25] F. Campanario, Q. Li, M. Rauch and M. Spira, ZZ+jet production via gluon fusion at the LHC, JHEP 1306 (2013) 069 [arXiv:1211.5429 [hep-ph]].

[26] F. Cascioli, T. Gehrmann, M. Grazzini, S. Kallweit, P. Maierhofer, A. von Manteuffel, S. Pozzorini, D. Rathlev et al., ZZ production at hadron colliders in NNLO QCD, Phys. Lett. B 735 (2014) 311 [arXiv:1405.2219 [hep-ph]].

[27] T. Gehrmann, M. Grazzini, S. Kallweit, P. Maierhofer, A. von Manteuffel, S. Pozzorini, D. Rathlev and L. Tancredi, $W^{+} W^{-}$Production at Hadron Colliders in Next to Next to Leading Order QCD, Phys. Rev. Lett. 113 (2014) 21, 212001 [arXiv:1408.5243 [hep-ph]].

[28] F. Caola and K. Melnikov, Constraining the Higgs boson width with ZZ production at the LHC, Phys. Rev. D 88 (2013) 054024 [arXiv:1307.4935 [hep-ph]].

[29] J. M. Campbell, R. K. Ellis and C. Williams, Bounding the Higgs width at the LHC using full analytic results for $g g \rightarrow e^{-} e^{+} \mu^{-} \mu^{+}$, JHEP 1404 (2014) 060 [arXiv:1311.3589 [hep-ph]]. 
[30] J. M. Campbell, R. K. Ellis and C. Williams, Bounding the Higgs width at the LHC: complementary results from $H \rightarrow W W$, Phys. Rev. D 89 (2014) 053011 [arXiv:1312.1628 [hep-ph]].

[31] J. M. Campbell, W. T. Giele and C. Williams, The Matrix Element Method at Next-to-Leading Order, JHEP 1211 (2012) 043 [arXiv:1204.4424 [hep-ph]].

[32] V. Khachatryan et al. (CMS Collaboration), Constraints on the Higgs boson width from off-shell production and decay to Z-boson pairs, Phys. Lett. B 736 (2014) 64 [arXiv:1405.3455 [hep-ex]].

[33] G. Aad et al. (ATLAS Collaboration), Determination of the off-shell Higgs boson signal strength in the high-mass ZZ and WW final states with the ATLAS detector, arXiv:1503.01060 [hep-ex].

[34] J. M. Campbell, R. K. Ellis, E. Furlan and R. Rontsch, Interference effects for Higgs boson mediated Z-pair plus jet production, Phys. Rev. D 90 (2014) 9, 093008 [arXiv:1409.1897 [hep-ph]].

[35] C. Englert and M. Spannowsky, Limitations and opportunities of off-shell coupling measurements, Phys. Rev. D 90 (2014) 053003 [arXiv: 1405.0285 [hep-ph]].

[36] C. Englert, Y. Soreq and M. Spannowsky, Off-shell Higgs coupling measurements in BSM scenarios, arXiv: 1410.5440 [hep-ph].

[37] H. E. Logan, Hiding a Higgs width enhancement from off-shell $g g\left(\rightarrow h^{*}\right) \rightarrow$ ZZ measurements, arXiv:1412.7577 [hep-ph].

[38] J. S. Gainer, J. Lykken, K. T. Matchev, S. Mrenna and M. Park, Beyond Geolocating: Constraining Higher Dimensional Operators in $H \rightarrow 4 \ell$ with Off-Shell Production and More, Phys. Rev. D 91 (2015) 3, 035011 [arXiv:1403.4951 [hep-ph]].

[39] M. Ghezzi, G. Passarino and S. Uccirati, Bounding the Higgs Width Using Effective Field Theory, PoS LL 2014 (2014) 072 [arXiv:1405.1925 [hep-ph]].

[40] G. Cacciapaglia, A. Deandrea, G. Drieu La Rochelle and J. B. Flament, Higgs couplings: disentangling New Physics with off-shell measurements, Phys. Rev. Lett. 113 (2014) 20, 201802 [arXiv:1406.1757 [hep-ph]].

[41] A. Azatov, C. Grojean, A. Paul and E. Salvioni, Taming the off-shell Higgs boson, Zh. Eksp. Teor. Fiz. 147 (2015) 410 [J. Exp. Theor. Phys. 120 (2015)] [arXiv:1406.6338 [hep-ph]].

[42] A. Biekoetter, A. Knochel, M. Kraemer, D. Liu and F. Riva, Vices and Virtues of Higgs EFTs at Large Energy, arXiv:1406.7320 [hep-ph].

[43] M. Buschmann, D. Goncalves, S. Kuttimalai, M. Schonherr, F. Krauss and T. Plehn, Mass effects in the Higgs-gluon coupling: boosted vs off-shell production, JHEP 1502 (2015) 038 [arXiv:1410.5806 [hep-ph]].

[44] L. J. Dixon and Y. Li, Bounding the Higgs Boson Width Through Interferometry, Phys. Rev. Lett. 111 (2013) 111802 [arXiv:1305.3854 [hep-ph]].

[45] S. P. Martin, Shift in the LHC Higgs diphoton mass peak from interference with background, Phys. Rev. D 86 (2012) 073016 [arXiv:1208.1533 [hep-ph]].

[46] D. de Florian, N. Fidanza, R. J. Hernandez-Pinto, J. Mazzitelli, Y. Rotstein Habarnau and G. F. R. Sborlini, A complete $O\left(\alpha_{s}^{2}\right)$ calculation of the signal-background interference for the Higgs diphoton decay channel, Eur. Phys. J. C 73 (2013) 4, 2387 [arXiv:1303.1397 [hep-ph]].

[47] S. P. Martin, Interference of Higgs diphoton signal and background in production with a jet at the LHC, Phys. Rev. D 88 (2013) 1, 013004 [arXiv:1303.3342 [hep-ph]]. 